\title{
COVERING THE BOUNDARY OF A CONVEX SET BY TILES
}

\author{
MAREK LASSAK
}

(Communicated by William J. Davis)

\begin{abstract}
Let Euclidean space $E^{n}$ be dissected by a finite family of hyperplanes. We estimate how many of the tiles obtained are sufficient to cover the boundary of a convex set $A \subset E^{n}$.
\end{abstract}

Let $P$ be a parallelogram divided into $\left(p_{1}+1\right)\left(p_{2}+1\right)$ smaller parallelograms by $p_{1}$ straight lines parallel to a pair of sides of $P$ and by $p_{2}$ straight lines parallel to the other. To cover the boundary of $P$ we need, of course, $2\left(p_{1}+p_{2}\right)$ of the smaller parallelograms. We show that the boundary of every convex set $A \subset P$ can be covered by no more than $2\left(p_{1}+p_{2}\right)$ of the smaller parallelograms. An analogous property holds for convex subsets of an $n$-dimensional parallelotope. We also give an estimate of a similar nature for the dissection of Euclidean $n$-space $E^{n}$ by a finite family of hyperplanes. The above estimates, and a more general one, are presented in Part 2 as corollaries of a monotonicity property formulated in the Theorem in Part 1. In Part 3, as an application, we present an estimate concerning Hadwiger's covering problem.

The boundary, the interior and the relative interior of a convex set $A \subset E^{n}$ are denoted by the symbols bd $A$, int $A$ and ri $A$, respectively. By a convex body we understand a compact convex set with nonempty interior.

1. A monotonicity property. Let us dissect the space $E^{n}$ by a finite family $\mathscr{H}$ of hyperplanes. We denote by $\mathscr{P}_{\mathscr{H}}$ the family of the tiles obtained, i.e. the family of all $n$-dimensional closed polyhedral sets whose boundary points, and only boundary points belong to $\bigcup \mathscr{H}$. Let $A$ be a convex set with nonempty interior. By $\mathscr{P}_{\mathscr{H}}(A)$ we denote the family of tiles of $\mathscr{P}_{\mathscr{H}}$ which have nonempty intersections with int $A$. Let $\mathscr{S}_{\mathscr{H}}(A)$ be the family of those sets of $\mathscr{P}_{\mathscr{H}}(A)$ which are unbounded or have a nonempty intersection with bd $A$. The symbol $s_{\mathscr{L}}(A)$ means the number of sets in $\mathscr{S}_{\mathscr{H}}(A)$.

THEOREM. If $\mathscr{H}$ is a finite family of hyperplanes and $A \subset B$ are convex sets in $E^{n}$ with nonempty interiors, then $s_{\mathscr{H}}(A) \leq s_{\mathscr{H}}(B)$.

ProOF. Since $s_{\mathscr{H}}$ of $A$ and of the closure of $A$ are equal, without loss of generality we can assume that $A$ and $B$ are closed. Take a point $c \in \operatorname{int} A$. Let $A_{\lambda}$ denote the intersection of $B$ and the homothetic copy of $A$ with center $c$ and ratio $\lambda>0$.

For every $D \in \mathscr{P}_{\mathscr{H}}$ such that $D \cap$ int $B \neq \varnothing$, the set of all $\lambda$ such that $D \in$ $\mathscr{S}_{\mathscr{H}}\left(A_{\lambda}\right)$ has the form of an interval $\{\lambda ; \alpha(D)<\lambda \leq \beta(D)\}$ if $D$ is bounded and contained in int $B$, or of an interval $\{\lambda ; \alpha(D)<\lambda\}$ in the opposite case. This

Received by the editors March 10, 1987 and, in revised form, July 23, 1987.

1980 Mathematics Subject Classification (1985 Revision). Primary 52A45. 
results from the monotonicity of the family $\left\{A_{\lambda} ; \lambda>0\right\}$ and from the fact that $D$ is a closed $n$-dimensional set.

Consider a bounded tile $K \in \mathscr{P}_{\mathscr{H}}$ contained in int $B$. It is clear that $K \cap$ bd $A_{\beta(K)} \neq \varnothing$. Therefore the convexity of $A_{\beta(K)}$ and the inclusion $K \subset A_{\beta(K)}$ imply that a vertex of $K$ lies in bd $A_{\beta(K)}$. Hence the family of faces of $K$ which are subsets of bd $A_{\beta(K)}$ is nonempty. Let $G$ be a maximal face of this kind. Clearly, $K$ and $G$ determine exactly one set $K(G) \in \mathscr{P}_{\mathscr{H}}$ which has $G$ as a face and lies on the opposite side (than $K$ ) of every hyperplane from $\mathscr{H}$ containing $G$.

Now, we show the equality

$$
K(G) \cap A_{\beta(K)}=G .
$$

Let $u \in K(G) \cap A_{\beta(K)}$. Suppose that $u \notin G$. Let $v \in \operatorname{ri} G$. Since $u \in K(G) \backslash G$ and since $G$ is a face of $K$, there exists $z \in K \backslash G$ such that $v$ belongs to the open segment joining $u$ and $z$. Let $w=(v+z) / 2$. There exists a face $F$ of $K$ such that $w \in \operatorname{ri} F$ (Theorem 18.2 of [8]). Obviously, $v, z \in F$. Thus $v \in \operatorname{ri} G$ implies $G \subset F$ (Theorem 18.1 of [8]), and $z \notin G$ implies $G \neq F$. We have $w \in K \subset A_{\beta(K)}$. If $w \in \operatorname{int} A_{\beta(K)}$, then in virtue of $u \in A_{\beta(K)}$ we obtain $v \in \operatorname{int} A_{\beta(K)}$ (Theorem 6.1 of [8]) in contradiction to $v \in G \subset \operatorname{bd} A_{\beta(K)}$. Therefore $w \in \operatorname{bd} A_{\beta(K)}$. Since $w \in \operatorname{ri} F \subset A_{\beta(K)}$, by Theorem 18.1 of [8] we get $F \subset$ bd $A_{\beta(K)}$, which contradicts the maximality of $G$. Thus $u \in G$. So $K(G) \cap A_{\beta(K)} \subset G$. The inverse inclusion is obvious, hence (1) is established.

From (1) and $G \subset$ bd $A_{\beta(K)}$ we get $K(G) \cap \operatorname{int} A_{\beta(K)}=\varnothing$. Moreover, from $K \subset$ int $B$ we see that $K(G) \cap \operatorname{int} A_{\lambda} \neq \varnothing$ for every $\lambda>\beta(K)$. Thus

$$
\alpha(K(G))=\beta(K) \text {. }
$$

If $K_{1}, K_{2} \in \mathscr{P}_{\mathscr{H}}$ are bounded and contained in int $B$, then

$$
K_{1} \neq K_{2} \text { implies } K_{1}\left(G_{1}\right) \neq K_{2}\left(G_{2}\right) \text {. }
$$

In the case $\beta\left(K_{1}\right) \neq \beta\left(K_{2}\right)$, this results from (2). Let $\beta\left(K_{1}\right)=\beta\left(K_{2}\right)$. Suppose, $K_{1}\left(G_{1}\right)=K_{2}\left(G_{2}\right)$. Applying (1) we get $G_{1}=G_{2}$ and consequently, $K_{1}=K_{2}$. Contradiction.

From (2) and (3) we see that the number $s_{\mathscr{H}}\left(A_{\lambda}\right)$ does not decrease when $\lambda$ increases. Since $\mathscr{H}$ is finite, for sufficiently great $\mu$ we have $s_{\mathscr{H}}(B)=s_{\mathscr{H}}\left(A_{\mu}\right) \geq$ $s_{\mathscr{H}}(A)$.

2. Estimates of the number of tiles covering the boundary of a convex set. Observe that

$$
\text { bd } A \subset \bigcup \mathscr{S}_{\mathscr{H}}(A) \text {. }
$$

Indeed, every point $a \in \mathrm{bd} A$ is the limit of a sequence of interior points of $A$, hence, by the finiteness of $\mathscr{P}_{\mathscr{H}}(A), a$ is the limit of a subsequence of points from a tile in $\mathscr{S}_{\mathscr{H}}(A)$, and consequently $a$ is an element of this tile. From (4) and Theorem we get

COROLLARY 1. Let a convex set $B \subset E^{n}$ with nonempty interior be dissected by a finite family $\mathscr{H}$ of hyperplanes. The boundary of every convex subset $A$ of $B$ can be covered with no more than $s_{\mathscr{X}}(B)$ of the tiles obtained.

When $A$ has nonempty interior, the implication is obvious. If the interior is empty, $A$ can be considered as a subset of the boundary of a convex set with nonempty interior. 
From Corollary 1 we obtain Corollaries 2 and 3.

COROLlaRY 2. Let $P \subset E^{n}$ be an n-dimensional parallelotope dissected into $\prod_{i=1}^{n}\left(p_{i}+1\right)$ smaller closed parallelotopes by $p_{1}+\cdots+p_{n}$ hyperplanes, where $p_{i}$ denotes the number of hyperplanes parallel to the ith pair of parallel $(n-1)$ dimensional faces of $P, i=1, \ldots, n$. The boundary of each convex set $A \subset P$ can be covered with no more than $\prod_{i=1}^{n}\left(p_{i}+1\right)-\prod_{i=1}^{n}\left(p_{i}-1\right)$ of the obtained smaller parallelotopes.

Let $u(\mathscr{H})$ denote the number of unbounded sets of $\mathscr{P}_{\mathscr{L}}$.

COROLLARY 3. The boundary of every convex set can be covered by no more than $u(\mathscr{H})$ tiles of $\mathscr{P}_{\mathscr{H}}$.

In $[10$, p. 61$]$, the reader can find a formula for $u(\mathscr{H})$ in terms of the lattice structure of the arrangement $\mathscr{H}$. Let us add an explicit estimate of $u(\mathscr{H})$ in $E^{n}$. The relation of parallelism divides $\mathscr{H}$ into subfamilies $\mathscr{H}_{k}, k=1, \ldots, m$, of parallel hyperplanes. Denote by $p_{k}$ the number of hyperplanes of $\mathscr{H}_{k}$ and by $L_{k}$ the hypersubspace parallel to them, $k=1, \ldots, m$. If $\bigcap_{k=1}^{m} L_{k} \neq\{0\}$, all tiles are unbounded. Assume that $\bigcap_{k=1}^{m} L_{k}=\{0\}$. Then $n \leq m$. For $0 \leq i \leq m$, let $\sigma_{i}=\sigma_{i}\left(p_{1}, \ldots, p_{m}\right)$ denote the $i$ th symmetric function of $p_{1}, \ldots, p_{m}$ (the sum of products $\prod_{t \in T} p_{t}$ over all $i$-element subsets $T$ of $\{1, \ldots, m\}$ if $i \neq 0$ and 1 if $i=0$ ). Subtracting the generalized versions of (4.1) and (4.2) as mentioned on p. 278 of [11] (or, equivalently, the formulas in Theorem 1 of [1] taking $r=d$ ) we get

$$
u(\mathscr{H}) \leq \sum_{j=1}^{n}\left(1-(-1)^{j}\right) \sigma_{n-j}
$$

with equality if the intersection of every $n$ hyperplanes from among $L_{1}, \ldots, L_{m}$ is $\{0\}$. Particularly, dissecting the plane by a family $\mathscr{H}$ of $s$ straight lines we obtain $u(\mathscr{H}) \leq 2 s$. More precisely, $u(\mathscr{H})=2 s$ if not all the lines are parallel $[9$, p. 351] and $u(\mathscr{H})=s+1$ if all the lines are parallel.

3. An estimate concerning Hadwiger's covering problem. Let $A \subset E^{n}$ be a convex body. Following $[4$, p. 276], by $L(A)$ we denote the smallest $t$ such that $A$ can be covered by $t$ homothetic copies of $A$, each with a positive ratio less than 1. Hadwiger [5] formulated the conjecture that $L(A) \leq 2^{n}$ for every convex body $A \subset E^{n}$. For $n=2$ the positive answer results from [7] but for $n \geq 3$ the problem remains open. Some estimates are known for every centrally symmetric convex body $M \subset E^{n}$ : the estimate $L(M) \geq 2^{n}(n \log n+n \log \log n+5 n)$ of Rogers (see [4, p. 284]), the estimate $L(M) \leq(n+1)^{n}$ of Levin and Petuhin (see [2, p. 45]) and $L(M) \leq 8$ for $n=3$ (see [6]).

It seems that no estimate of $L(A)$ has been published for an arbitrary convex body $A \subset E^{n}$. Our purpose is to give such a one.

Let $h_{\alpha}^{c}$ denote the homothety with center $c$ and ratio $\alpha$. It is easy to check that

$$
h_{\alpha}^{c}(A) \cap h_{\gamma}^{c}(A) \subset h_{\beta}^{c}(A)
$$

for every $c \in E^{n}$ and $0 \leq \alpha \leq \beta \leq \gamma$. Let bd $A \subset \bigcup_{i=1}^{k} h_{\alpha_{i}}^{c_{i}}(A)$, where $0<\alpha_{i}<1$ for $i=1, \ldots, k$. Take an $x \in \operatorname{int} A$ and positive $\beta_{i}$ such that $x \in h_{\beta_{i}}^{c_{i}}(A)$ for 
$i=1, \ldots, k$. From (5) we see that $A \subset \bigcup_{i=1}^{k} h_{\beta_{i}}^{c_{i}}(A)$. Consequently,

$L(A)$ is equal to the smallest number of homothetic copies of $A$, each with a positive ratio less than 1 , which cover bd $A$.

There is a parallelotope $P$ containing $A$ such that some homothetic copy $(1 / n) P$ of $P$ of ratio $1 / n$ is contained in $A$ (see [3]). So a copy $(1 /(n+1)) P$ is a subset of $(n /(n+1)) A$. Since $n /(n+1)<1$, from Corollary 2 and (6) we get that

$$
L(A) \leq(n+1)^{n}-(n-1)^{n} .
$$

This inequality can be improved.

COROLlaRY 4. For every convex body $A \subset E^{n}$ we have

$$
L(A) \leq(n+1) n^{n-1}-(n-1)(n-2)^{n-1} .
$$

For the proof consider a parallelotope $P$ of the smallest volume containing $A$. It has the form of a translate of the set $\left\{\sum_{i=1}^{n} \alpha_{i} a_{i} ; 0 \leq \alpha_{i} \leq 1\right.$ for $\left.i=1, \ldots, n\right\}$, where $a_{1}, \ldots, a_{n}$ are independent vectors. Repeating the consideration of the proof of Lemma 1 of [3] we see that a translate of the set $\left\{\sum_{i=1}^{n} \lambda_{i} \alpha_{i} a_{i} ; 0 \leq \alpha_{i} \leq 1\right.$ for $i=1, \ldots, n\}$, where $\lambda_{1}, \ldots, \lambda_{n}$ are positive numbers, is contained in a homothetic copy of $A$ with ratio $\sum_{i=1}^{n} \lambda_{i}$. Put $\lambda_{1}=\cdots=\lambda_{n-1}=1 / n$ and $\lambda_{n}=1 /(n+1)$. Now, $\sum_{i=1}^{n} \lambda_{i}<1$. From Corollary 2 and (6) we obtain the promised inequality.

\section{REFERENCES}

1. G. L. Alexanderson and J. E. Wetzel, Division of space by parallels, Trans. Amer. Math. Soc. 291 (1985), 363-377.

2. V. G. Boltjansky and I. Ts. Gohberg, Results and problems in combinatorial geometry, Cambridge Univ. Press, Cambridge, 1985.

3. G. D. Chakerian and S. K. Stein, Some intersection properties of convex bodies, Proc. Amer. Math. Soc. 18 (1967), 109-112.

4. B. Grünbaum, Borsuk's problem and related questions, Convexity, Proc. Sympos. Pure Math., vol. 7, Amer. Math. Soc., Providence, R. I., 1963, pp. 271-284.

5. H. Hadwiger, Ungeloste Probleme. Nr 20, Elem. Math. 12 (1957), 121.

6. M. Lassak, Solution of Hadwiger's covering problem for centrally symmetric convex bodies in $E^{3}$, J. London Math. Soc. (2) 30 (1984), 501-511.

7. F. W. Levi, Überdeckung eines Eibereiches durch Parallelverschiebungen seines offenen Kerns, Arch. Math. 6 (1955), 369-370.

8. R. T. Rockafellar, Convex analysis, Princeton Univ. Press, Princeton, N. J., 1970.

9. J. Steiner, Einige Gesetze über die Theilung der Ebene und des Raumes, J. Reine Angew. Math. 1 (1826), 349-364.

10. T. Zaslavsky, Facing up to arrangements, Mem. Amer. Math. Soc. no. 154 (1975).

11. _ , A combinatorial analysis of topological dissections, Adv. in Math. 25 (1977), 267-285.

Department of Mathematics, University of Washington, Seattle, WashingTON 98195

Permanent address: Instytut Matematyki i Fizyki ATR, 85-790 Bydgoszcz, Poland 